\title{
Optical micro-spectroscopy of single metallic nanoparticles: quantitative extinction and transient resonant four- wave mixing $\dagger$
}

\author{
Lukas Payne, ${ }^{a}$ George Zoriniants, ${ }^{a}$ Francesco Masia, ${ }^{b}$ Kenton P. Arkill, ${ }^{c}$ \\ Paul Verkade, ${ }^{c}$ Darren Rowles, ${ }^{d}$ Wolfgang Langbein ${ }^{b}$ and Paola Borri*a
}

Received 14th May 2015, Accepted 29th May 2015

DOI: $10.1039 / c 5 f d 00079 c$

We report a wide-field imaging method to rapidly and quantitatively measure the optical extinction cross-section $\sigma_{\text {ext }}$ (also polarisation resolved) of a large number of individual gold nanoparticles, for statistically-relevant single particle analysis. We demonstrate a sensitivity of $5 \mathrm{~nm}^{2}$ in $\sigma_{\text {ext }}$, enabling detection of single $5 \mathrm{~nm}$ gold nanoparticles with total acquisition times in the 1 min range. Moreover, we have developed an analytical model of the polarisation resolved $\sigma_{\text {ext }}$ which enabled us to extract geometrical particle aspect ratios from the measured $\sigma_{\text {ext. }}$ Using this method, we have characterized a large number of nominally-spherical gold nanoparticles in the 10-100 nm size range. Furthermore, the method provided measurements of in-house fabricated nanoparticle conjugates, allowing distinction of individual dimers from single particles and larger aggregates. The same particle conjugates were investigated correlatively by phaseresolved transient resonant four-wave mixing micro-spectroscopy. A direct comparison of the phase-resolved response between single gold nanoparticles and dimers highlighted the promise of the four-wave mixing technique for sensing applications with dimers as plasmon rulers.

\section{Introduction}

In the last decade, increasing attention has been devoted to methods for the optical detection of single nano-objects, which do not rely on the object's fluorescence emission, but on its absorption and scattering properties (for a recent review see ref. 1). These characteristics are particularly relevant for weakly

${ }^{a}$ Cardiff University School of Biosciences, Museum Avenue, Cardiff CF10 3AX, United Kingdom. E-mail: borrip@cf.ac.uk

${ }^{b}$ Cardiff University School of Physics and Astronomy, The Parade, Cardiff CF24 3AA, United Kingdom ${ }^{c}$ University of Bristol, Wolfson Bioimaging Facility, University Walk, Bristol BS8 1TD, United Kingdom ${ }^{d}$ BBI Solutions OEM Ltd, Golden Gate, Ty-Glas Avenue, Llanishen, Cardiff CF14 5DX, United Kingdom $\dagger$ Electronic supplementary information (ESI) available. See DOI: 10.1039/c5fd00079c 
luminescent systems, such as metallic nanoparticles (NPs), whose morphologydependent localized surface plasmon resonances (LSPR) produce large particle polarisabilities and, in turn, large absorption and scattering cross-sections at the LSPR frequency. Notably, these resonances can be exploited to image metallic NPs with high sensitivity, and to probe nanoscale regions in the NP vicinity via the local field enhancement effect. Possible applications range from photonics devices, ${ }^{2}$ catalysis ${ }^{3}$ and photovoltaics ${ }^{4}$ to biomedical imaging, ${ }^{5,6}$ sensing ${ }^{7-9}$ and medical therapy. ${ }^{\mathbf{1 0}}$ For many of these applications, it is essential to have a quantitative knowledge of the linear and nonlinear NP optical properties at the single particle level, beyond traditional ensemble-averaged measurements.

A number of approaches have been developed recently to measure the optical extinction cross-section $\sigma_{\text {ext }}$ (defined as $\sigma_{\text {ext }}=P_{\text {ext }} / I_{\mathrm{i}}$ with $I_{\mathrm{i}}$ the incident intensity and $P_{\text {ext }}$ the total power lost, due to both absorption and scattering) of single NPs, and to separate this into the absorption and the scattering cross-section, via $\sigma_{\text {ext }}=\sigma_{\text {abs }}+\sigma_{\text {sca }}$. The scattering cross-section $\sigma_{\text {sca }}$ is most commonly measured using dark-field microscopy, which offers the advantage of a wide-field illumination with commercial easy-to-use optical set-ups and detector cameras and, in turn, the rapid observation of a large number of individual NPs simultaneously for statistical analysis. However, the quantification of $\sigma_{\text {sca }}$ in absolute units in this technique is usually not reported, since it requires the detailed knowledge of the excitation and collection geometries, or a calibration reference. Moreover, for particles much smaller than the wavelength of light (dipole approximation), $\sigma_{\text {sca }}$ decreases with the square of the particle volume, limiting the practical applicability of dark-field microscopy to NPs of moderately large sizes (>20 nm diameter for gold and silver nanospheres ${ }^{1}$ ).

A sensitive quantitative method to measure $\sigma_{\text {ext }}$ uses a spatial modulation micro-spectroscopy (SMS) technique. ${ }^{11}$ The method was shown to achieve detection of single gold NPs as small as $5 \mathrm{~nm}$ in diameter, corresponding to a sensitivity in terms of $\sigma_{\text {ext }}$ down to about $5 \mathrm{~nm}^{2}$. However, for quantitative determination of $\sigma_{\text {ext }}$ this method needs a precise knowledge of the modulation amplitude and beam profile at the sample. Moreover it is a modulation-based laser-scanning technique, therefore less amenable to the rapid characterization of a large number of NPs compared to wide-field techniques. Additionally, it requires rather costly and specialized equipment. Similar considerations hold for photothermal imaging (PI), ${ }^{12}$ another laser-scanning modulation-based technique. Moreover, in PI quantifying the absorption cross-section amplitude in absolute units is not trivial, since absorption is only indirectly detected in photothermal signals through the temperature change in the surrounding medium following NP heating.

We have recently shown a simple wide-field microscopy method with which we measured $\sigma_{\text {ext }}$ on a large number $(>100)$ of single gold NPs rapidly, quantitatively in absolute units, and without the need for calibration standards or complex modulation techniques. ${ }^{13}$ In this first demonstration, we showed measurements on gold NPs of $40 \mathrm{~nm}$ and $100 \mathrm{~nm}$ diameter for proof-of-principle, and a sensitivity in $\sigma_{\text {ext }}$ of around $500 \mathrm{~nm}^{2}$, limited by the acquisition speed of the consumer color camera used for detection. We also showed that we could characterize the NP asphericity by using linearly polarized illumination, however the measured polarisation dependence was not analyzed quantitatively to extract the geometrical aspect ratio of the NP. 
Beyond linear optical properties, we also developed a nonlinear optical technique, which detects single gold NPs via their transient degenerate four-wave mixing (FWM) resonant at the LSPR., ${ }^{6,14}$ Owing to an interferometric and timeresolved detection, this technique is specific to gold NPs and background-free (contrary to the above mentioned methods), even in highly scattering and fluorescing environments, such as cells and tissue. Notably, rather than providing the absolute value of $\sigma_{\text {ext }}$, the technique is sensitive to the change in the NP extinction induced by a short pump pulse at the LSPR. In turn, owing to the phase-sensitivity of the interferometric detection, we showed that we could measure the pumpinduced ultrafast change in the particle polarisability as a complex quantity, i.e. separating its real and imaginary parts. ${ }^{14}$ The phase of this complex quantity was shown to be sensitive to the relative shift between the LSPR and the optical wavelength in the FWM experiment. Hence, beyond fundamental interest, this background-free, phase-resolved, intrinsic ratiometric readout, at the singleparticle level, could be exploited for LSPR-based sensing applications, for example using NP dimers as plasmon rulers. ${ }^{9}$

In this work, we report a major improvement on our previous wide-field extinction microscopy method, which now enables us to reach a sensitivity in $\sigma_{\text {ext }}$ of about $5 \mathrm{~nm}^{2}$, hence allowing detection of single $5 \mathrm{~nm}$ diameter gold NPs. The technique remains rapid, wide-field, and easy to implement on any conventional microscope. We also present a method to extract the nanoparticle geometrical aspect ratio from the measured polarisation-resolved $\sigma_{\text {ext }}$ using analytical formulas for elliptical particles. With this wide-field extinction technique, we have characterized a statistically significant number of gold NP conjugates fabricated in house, and used the measurements to distinguish single dimers from isolated particles and larger aggregates. We then performed FWM measurements on the same NP conjugates, allowing direct correlation with the optical extinction method. The results highlighted the promise of the FWM technique for sensing applications.

\section{Wide-field extinction microscopy}

\subsection{Isolated quasi-spherical nanoparticles}

The experimental set-up consists of an inverted microscope (Nikon Ti-U) equipped with a white-light illumination (halogen lamp $100 \mathrm{~W}$ with Nikon neutral color balance filter or light balancing LB200 optical blue Hoya filter), an oil condenser of 1.4 numerical aperture (NA) with a removable home-built dark-field illumination of 1.1-1.4 NA, a choice between a $40 \times 0.95$ NA dry objective and a $100 \times$ 1.45 NA oil objective, $1 \times$ or $1.5 \times$ intermediate (tube lens) magnification, a consumer Canon EOS 40D color camera attached to the left port of the microscope and the PCO Edge 5.5, a low noise, high frame-rate, grayscale (b/w), watercooled, scientific-CMOS camera (sCMOS), attached to the eye-piece side port. With the Canon camera, images were taken in Canon 14-bit RAW format with 10.1 megapixel resolution. The RAW images were converted using the DCRAW plugin in ImageJ, providing 16 bit RGB images with a linear response to intensity and no color balancing. The color camera enables a coarse spectroscopic detection, separating the three wavelength ranges of red (R) 570-650 nm, green (G) 480-580 $\mathrm{nm}$, and blue (B) 420-510 $\mathrm{nm}$. With the sCMOS camera, spectroscopic detection was achieved using bandpass filters (Semrock) in the excitation beam path, in the 
wavelength ranges of $470 \pm 11 \mathrm{~nm}$ for blue, $530 \pm 20 \mathrm{~nm}$ for green and $607 \pm 18$ $\mathrm{nm}$ for red.

The investigated samples were nominally spherical gold NPs of $10 \mathrm{~nm}, 30 \mathrm{~nm}$, $40 \mathrm{~nm}, 60 \mathrm{~nm}, 80 \mathrm{~nm}$ and $100 \mathrm{~nm}$ diameter (BBI Solutions) drop cast onto a glass coverslip, covered in silicone oil (refractive index $n=1.518$ ) and sealed with a glass slide. Before use, glass slides and coverslips were cleaned thoroughly to avoid debris. Cleaning was performed first with acetone and high-quality cleanroom wipes, followed by a chemical etch called Caro's etch, or more commonly, Piranha etch.

Extinction microscopy was performed as described in ref. 13. Briefly, the condenser NA was adjusted to match that of the objective. Two bright-field images were taken; one with the NPs in focus, and another with the NPs either out-offocus (moving the objective by approximately $d=15 \mu \mathrm{m}$ axially away from the sample) or laterally shifted by approximately $1 \mu \mathrm{m}$. Background images were taken for blocked illumination. To achieve the lowest shot noise with the Canon camera, the lowest camera sensitivity was used (100 ISO), for which the full-well capacity of the pixels of about $N_{\mathrm{fw}}=4 \times 10^{4}$ electrons occurs at $70 \%$ of the digitizer range ( 3.4 electrons per count). An exposure time in the order of $10 \mathrm{~ms}$ was chosen to reach $N_{\mathrm{fw}}$. Averaging over $N_{\mathrm{a}}=36$ acquisitions was performed for each image set. With the sCMOS, we had $N_{\mathrm{fw}}=30000$ electrons ( 0.54 electrons per count, 16 bit dynamic range), an exposure time of $1 \mathrm{~ms}$ and $N_{\mathrm{a}}=25600$ at 400 frames per s, resulting in a total acquisition time of $64 \mathrm{~s}$ for an image set.

We call the background-subtracted, transmitted intensity of the bright-field image with NPs in focus $I_{\mathrm{f}}$, and the defocused, or shifted, intensity $I_{0}$. In the defocused image, a NP distributes its effect over a radius of about $r_{\mathrm{d}}=\mathrm{NA} d$, making $I_{0}$ similar to the intensity $I_{\mathrm{f}}$ in the absence of the NP. In the shifted image, we directly measure the intensity in the absence of the NP, which has been sideways shifted from its location. The extinction cross-section of a NP located within the area $A_{\mathrm{i}}$ in the image can then be expressed as $\sigma_{\text {ext }}=\int_{A_{\mathrm{i}}} \Delta \mathrm{d} A$, with the relative extinction $\Delta=\left(I_{0}-I_{\mathrm{f}}\right) / I_{0}$. To account for the slight mismatch between $I_{0}$ and $I_{\mathrm{f}}$ without a NP due to, for example, a drift of the illumination intensity, the residual influence of the NP out-of-focus, and/or a non-perfect optical quality of the glass surface, we determine a local background extinction $\Delta_{\mathrm{b}}=A_{\mathrm{b}}{ }^{-1} \int_{A_{\mathrm{b}}} \Delta \mathrm{d} A$ in the area $A_{\mathrm{b}}$ between the radius $r_{\mathrm{i}}$ and $2 r_{\mathrm{i}}$, yielding the background-corrected $\sigma_{\text {ext }}=\int_{A_{\mathrm{i}}}\left(\Delta-\Delta_{\mathrm{b}}\right) \mathrm{d} A$. As discussed in ref. 13, a good choice of $r_{\mathrm{i}}$, for which saturation of $\sigma_{\text {ext }}$ is reached, is given by $\sim 3 \lambda /(2 \mathrm{NA})$, approximately at the second Airy ring of the objective point-spread function.

In order to determine $\sigma_{\text {ext }}$ of many particles from an extinction image, we developed an image analysis programme written in ImageJ macro language, as described in ref. 13 (updated version available). Isolated spherical NPs are distinguished using their extinction color. NPs with $\sigma_{\text {ext }}$ largest in the color channel corresponding to the expected plasmon resonance are retained, i.e. green (red) for 10-40 nm (100 nm) spherical gold NPs having a LSPR dominated by the absorption (scattering) part of the extinction at around $540 \mathrm{~nm}(\sim 590 \mathrm{~nm})$ in a surrounding medium of 1.5 refractive index. NPs which likely correspond to 
aggregates, debris, or largely non-spherical NPs are excluded in this way. NPs of $60 \mathrm{~nm}$ and $80 \mathrm{~nm}$ nominal diameter have a size range distribution which allows for isolated particles to have a $\sigma_{\text {ext }}$ largest in either the green or the red channel of the Canon camera. This was taken into account in the image analysis automated routine. The statistical results over 54 individual gold NPs of nominally $10 \mathrm{~nm}$ diameter are summarised in Fig. 1, measured using the $100 \times 1.45$ NA oil objective, the sCMOS camera, and by laterally shifting the sample for reference. An example of an extinction image is shown in Fig. 1a, demonstrating the high contrast in visualizing single $10 \mathrm{~nm}$ NPs (which appear as two spots, one bright and one dark, due to the lateral shifting). We adopted a "time-symmetric referencing" procedure, namely $I_{0}$ was measured before and after $I_{\mathrm{f}}$ and its average value was used for reference. This reduces the effect of slow drifts. The distribution of $\sigma_{\text {ext }}$ in the $\mathrm{G}$ channel corresponding to the plasmon resonance has a mean of $\bar{\sigma}_{\text {ext }}=69 \mathrm{~nm}^{2}$, which is consistent with experimental and theoretical values found in the literature. ${ }^{15,16}$ The measurement noise in the experiment, $\hat{\sigma}_{\text {noise }}$, is obtained from the standard deviation of the zero-mean distribution of $\sigma_{\text {ext }}$ in image regions free of NPs. As shown in Fig. 1, we find $\hat{\sigma}_{\text {noise }}=5.9 \mathrm{~nm}^{2}$ for

a)
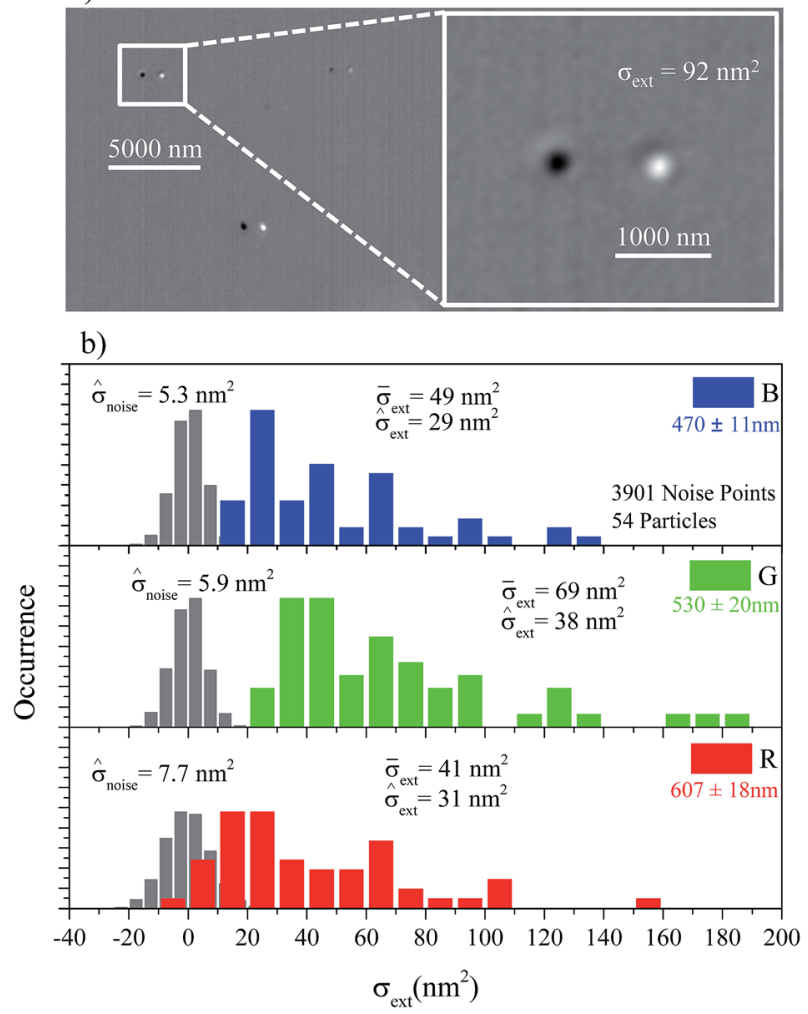

Fig. 1 (a) Wide-field extinction image of single gold NPs of nominal $10 \mathrm{~nm}$ diameter in the G channel, using a $100 \times 1.45$ NA oil objective and a sCMOS camera (see text). The right panel is a zoom over the region indicated by the white frame. Grey scale $\min =-0.005$, $\max =0.00055$. (b) Distributions of $\sigma_{\text {ext }}$ for 54 gold NPs of nominal $10 \mathrm{~nm}$ diameter in the $R, G$, and $B$ color channels as indicated. The grey histograms show the noise distribution obtained by measuring $\sigma_{\text {ext }}$ in 3901 randomly selected regions without NPs. 
the green channel, and down to $\hat{\sigma}_{\text {noise }}=5.3 \mathrm{~nm}^{2}$ in the blue channel. Notably, this number is two orders of magnitude lower than what was shown in our previous work using the consumer Canon camera. The main reason for such reduction of $\hat{\sigma}_{\text {noise }}$ is the large number of averages $\left(N_{\mathrm{a}}=25600\right)$ possible with the high-speed sCMOS camera, while retaining a practically feasible total acquisition time over which setup drifts are acceptable. The experimental $\hat{\sigma}_{\text {noise }}$ is, however, still larger than the theoretical shot-noise limit of $3 \mathrm{~nm}^{2}$, as estimated using the expression in ref. 13. This is likely due to a non-perfect referencing in the measurement of the extinction image $\Delta$, namely $I_{0}$ not being identical to the intensity $I_{\mathrm{f}}$ in the absence of the NP, even with local background correction. To that end, we find that defocusing the objective gives $\hat{\sigma}_{\text {noise }}=25 \mathrm{~nm}^{2}$ compared to $\hat{\sigma}_{\text {noise }}=5.9 \mathrm{~nm}^{2}$ when laterally shifting the sample (under otherwise identical conditions), most likely because a lateral shift does not alter the beam path geometry and thus enables a better referencing. We also find $\hat{\sigma}_{\text {noise }}=7 \mathrm{~nm}^{2}$ when laterally shifting but without time symmetric referencing, indicating that sample/objective/camera drifts play a role. Furthermore, at this sensitivity, the spatial and optical quality of the glass surface provides a spatial extinction structure which contributes to $\hat{\sigma}_{\text {noise }}$.

In the histogram in Fig. 1, the standard deviation of $\sigma_{\text {ext }}$ is much larger than $\hat{\sigma}_{\text {noise }}$ and is dominated by the NP size/shape/environment distribution. The NP-related standard deviation, $\hat{\sigma}_{\text {ext }}$, is determined using $\hat{\sigma}_{\text {ext }}{ }^{2}=\hat{\sigma}_{\text {total }}{ }^{2}-\hat{\sigma}_{\text {noise }}{ }^{2}$, where $\hat{\sigma}_{\text {total }}$ is the total standard deviation of $\sigma_{\text {ext }}$ as measured. Using $\delta R / R=\hat{\sigma}_{\text {ext }} /\left(3 \bar{\sigma}_{\text {ext }}\right)$, we then inferred a size distribution $\delta R / R=0.18$ ( $R$ is the NP radius). This value is larger than the manufacturer specified distribution $\delta R / R=0.10$, as determined by electron microscopy. This suggests, consistent with the literature, that additional factors influence the optical $\hat{\sigma}_{\text {ext }}$, beyond size fluctuations, such as the NP asphericity, ${ }^{17}$ fluctuations in the local dielectric environment, and the electron-surface scattering damping parameter. ${ }^{16}$

An overview of $\bar{\sigma}_{\text {ext }}$ and $\hat{\sigma}_{\text {ext }}$ in the green color channel for NPs in the 10-60 nm diameter range, and both green and red channels for $60 \mathrm{~nm}, 80 \mathrm{~nm}$ and $100 \mathrm{~nm}$ sized NPs, is shown in Fig. 2. Measurements for NPs in the $30-100 \mathrm{~nm}$ size range were performed using the $40 \times 0.95$ NA objective, 1.5 magnifier and the consumer Canon camera, as described in ref. 13. For each nominal diameter, a statistical analysis on $>50$ individual gold NPs was performed. The bars indicate the standard deviation $\hat{\sigma}_{\text {ext }}$. We also measured $\sigma_{\text {sca }}$ using dark-field, as described in ref. 13, and in turn deduced $\sigma_{\mathrm{abs}}$ from $\sigma_{\text {ext }}$, as shown in Fig. 2. Note that for these data the calibration constant $\eta$ (such that $\sigma_{\mathrm{sca}}=\eta \int_{A_{\mathrm{i}}} I_{\mathrm{df}} \frac{\mathrm{d} A}{I_{0}}$ with $I_{\mathrm{df}}$ scattered intensity in dark-field) was measured by using nanodiamonds, ${ }^{18}$ which are scattering-only particles, where $\sigma_{\text {sca }}=\sigma_{\text {ext }}$. A comparison between the experimental values and calculations from Mie theory ${ }^{15}$ of $\sigma_{\text {sca }}$ and $\sigma_{\text {abs }}$ at the LSPR wavelength is shown in Fig. 2, indicating good agreement (note how for the largest particles the scattering cross-section in the $\mathrm{R}$ channel dominates). Deviations between experiment and theory could be due to the spectral averaging of the color channel bandwidth, especially when using the consumer Canon camera. Furthermore, as mentioned in ref. 13, the finite angular range of the objective implies an underestimate of the extinction. In Fig. 2 we also compare $\hat{\sigma}_{\text {ext }}$ with the manufacturer specified size distribution (black error bars) using $\delta R / R=\hat{\sigma}_{\text {ext }} /\left(\bar{\sigma}_{\text {ext }} \gamma\right)$ with $\gamma=3$ for small particles, where the extinction is dominated by absorption. For particle sizes 


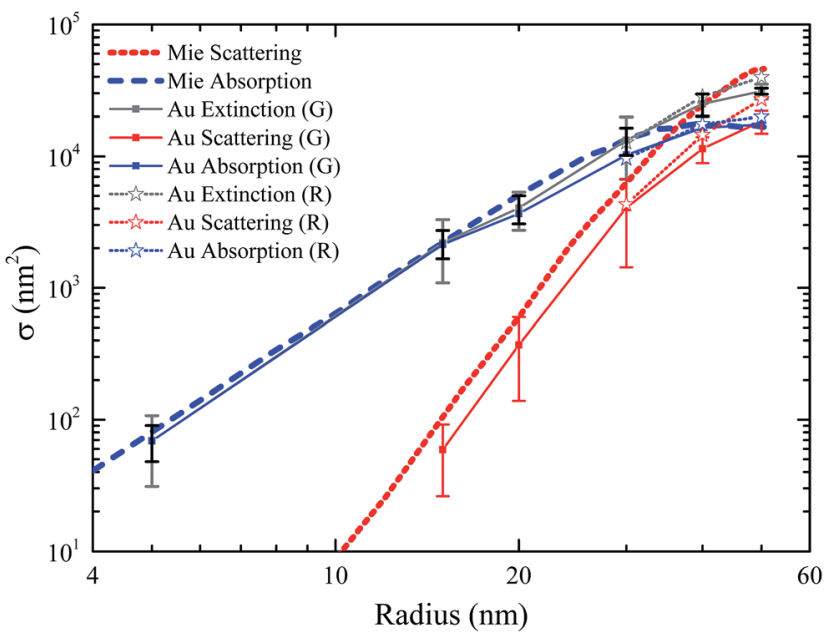

Fig. 2 Extinction, scattering and absorption cross section measured in the $G$ channel on a series of nominally spherical gold NPs with a nominal diameter from $10 \mathrm{~nm}$ to $100 \mathrm{~nm}$ (solid squares). For $60 \mathrm{~nm}, 80 \mathrm{~nm}$ and $100 \mathrm{~nm}$ diameters, the values measured in the R channel are also given (empty stars). The thick blue dashed (red dotted) line is the calculated absorption (scattering) cross section from Mie theory at the LSPR. Symbols (connected by thin lines as a guide to the eye) give the experimental cross-sections as an average over $>50$ individually measured NPs in the wide-field image, and bars give the standard deviation due to NP size/shape/environment distribution. Black bars are the manufacturer specified size distributions (see text). Note that $10 \mathrm{~nm}$ gold NPs cannot be measured in dark-field due to their small $\sigma_{\text {sca. }}$.

larger than $40 \mathrm{~nm}$ diameter, $\gamma$, such that $\sigma_{\text {ext }} \propto R^{\gamma}$, was extracted from $\sigma_{\text {ext }}$ calculated from Mie theory using the sum of $\sigma_{\text {sca }}$ and $\sigma_{\text {abs }}$ at the corresponding LSPR wavelength. We find that $\hat{\sigma}_{\text {ext }}$ is generally larger than the manufacturer specifications, again suggesting that additional factors influence the optical $\hat{\sigma}_{\text {ext }}$, beyond size fluctuations.

\subsection{Nanoparticle asphericity}

In our previous work, we showed that by measuring the dependence of $\sigma_{\text {ext }}$ on the in-plane linear polarisation angle $\theta$ of the exciting light we could extract information on the NP asphericity. ${ }^{13}$ In particular, we fitted the experimental $\sigma_{\text {ext }}$ with the expression $\sigma_{\text {ext }}(\theta)=\sigma_{0}\left(1+\alpha_{\mathrm{P}} \cos \left(2\left(\theta-\theta_{0}\right)\right)\right)$, where $\sigma_{0}$ is the polarisationaveraged $\sigma_{\text {ext }}, \alpha_{\mathrm{P}} \geq 0$ is the amplitude of the polarisation dependence, and $0 \geq \theta_{0}$ $\geq \pi$ is an angular offset, indicating the orientation (in-plane) of the NP asymmetry. We found that, within the measurement noise, aspherical NPs could be identified as those having $\alpha_{\mathrm{P}}$ larger than about 0.1 (in the red channel). However, the relationship between $\alpha_{\mathrm{P}}$ and the particle aspect-ratio was not derived, hence this parameter remained a phenomenological value rather than a useful structural quantity. Here we have developed a theoretical model for aspherical particles, with which we have simulated $\sigma_{\text {ext }}(\theta)$ and, in turn, calculated $\alpha_{\mathrm{P}}$ as a function of the particle aspect ratio.

We describe a non-spherical particle as a metallic ellipsoid with three orthogonal semi-axes $a, b$ and $c$. In the particle reference system, the polarisability 
tensor $\hat{\alpha}$ is diagonal, and its eigenvalues (in the limit of particles with a radius much smaller than the light wavelength) are given by ${ }^{\mathbf{1 9}}$ $\alpha_{i}=4 \pi a b c \frac{\varepsilon-\varepsilon_{\mathrm{m}}}{3 \varepsilon_{\mathrm{m}}+3 L_{i}\left(\varepsilon-\varepsilon_{\mathrm{m}}\right)} . \varepsilon$ is the dielectric constant of gold, $\varepsilon_{\mathrm{m}}$ is the dielectric constant of the medium surrounding the NP, and $L_{i}$ with $i=a, b, c$ are dimensionless quantities defined by the particle geometry (see ESI $\dagger$ ). We then expressed $\sigma_{\text {ext }}$ as a function of $\hat{\alpha}$, taking into account that for an arbitrary particle orientation in the laboratory system, the polarisability tensor is $\hat{\alpha}=\hat{A}^{-1} \hat{\alpha}^{\prime} \hat{A}$, where $\hat{A}$ is the rotation matrix transforming the particle reference system into the laboratory system, and $\alpha^{\prime}$ is the polarisability tensor in the laboratory system. The absorption, scattering, and extinction cross-sections are calculated following ref. 19 (for more details see ESI $\dagger$ ). If the polarisation of light is parallel to one of the symmetry axes of the particle, the extinction cross-section is given by:

$$
\sigma_{\mathrm{ext}, m}=k \Im\left(\alpha_{m}\right)+\frac{k^{4}\left|\alpha_{m}\right|^{2}}{6 \pi},
$$

where $m=1,2,3$ and $k=2 \pi n / \lambda$ with $\lambda$ the wavelength in vacuum, $n$ is the refractive index of the surrounding medium, and $\mathfrak{s}$ denotes the imaginary part. If the particle is oriented in-plane at an angle $\gamma$ with respect to the laboratory $x$-axis, the extinction cross-section for an incident polarized light in-plane at an angle $\theta$ with respect to the laboratory $x$-axis can be accordingly calculated using the rotation matrix $\hat{A}$, and is found to be (for details see ESI $\dagger$ ):

$$
\sigma_{\text {ext }}(\theta)=\sigma_{\text {ext }, 1} \cos ^{2}(\gamma-\theta)+\sigma_{\text {ext }, 2} \sin ^{2}(\gamma-\theta)
$$

This represents a sinusoidal dependence of the measured extinction crosssection versus $\theta$, which can be rewritten as:

$$
\sigma_{\mathrm{ext}}(\theta)=\bar{\sigma}\left(1+\alpha_{\mathrm{P}} \cos [2(\theta-\gamma)]\right)
$$

with

$$
\bar{\sigma}=\frac{\sigma_{\mathrm{ext}, 1}+\sigma_{\mathrm{ext}, 2}}{2}, \quad \alpha_{\mathrm{P}}=\frac{\sigma_{\mathrm{ext}, 1}-\sigma_{\mathrm{ext}, 2}}{\sigma_{\mathrm{ext}, 1}+\sigma_{\mathrm{ext}, 2}}
$$

With these formulas, for a non-spherical particle with in-plane asymmetry $a>$ $b$ (note that in the experiment we are not sensitive to the asymmetry in the $z$-axis), we calculated $\alpha_{\mathrm{P}}$ and $\bar{\sigma}$ as a function of the particle ellipticity parameter $1-b / a$ and the particle volume $(4 / 3) \pi a b c$ (where we used $c=\sqrt{a b}$ ). We find that in the limit of small ellipticity $\left(\alpha_{\mathrm{P}}<0.5\right), \alpha_{\mathrm{P}}$ is proportional to the ellipticity parameter and is almost independent of the particle volume (see ESI $\dagger$ ). Thus, the ellipticity can be simply determined as $1-b / a=K \alpha_{\mathrm{P}}$ with the parameter $K$ being a function of the wavelength range used in the experiment. For the $\mathrm{G}$ and $\mathrm{R}$ channels of the Canon $40 \mathrm{D}$ camera used in ref. 13 , we find $K_{\mathrm{G}}=0.568$ and $K_{\mathrm{R}}=0.339$. Therefore a measured $\alpha_{\mathrm{P}}=0.1$ in the R channel corresponds to an ellipticity $1-b / a$ of $3.39 \%$.

\subsection{Nanoparticle dimers}

We investigated the applicability of this wide-field imaging method to the characterization of gold NP dimers fabricated in-house. Nominally spherical $40 \mathrm{~nm}$ 
diameter gold NPs coated with a nominal $6 \mathrm{~nm}$ thick silica shell were fabricated by BBI Solutions. We covalently bound them to bare $40 \mathrm{~nm}$ gold NPs in solution using (3-mercaptopropyl)trimethoxysilane (3-MT). Briefly, this was achieved by mixing equal volumes ( $250 \mu \mathrm{l}$ each) of bare and silica-coated NPs stock solutions (each at a concentration of approximately $9 \times 10^{9} \mathrm{NP}$ per ml) with $0.985 \mathrm{ml}$ of water (Millipore) and $15 \mu \mathrm{l}$ of 3-MT. The dimerisation reaction was monitored in real-time through the occurrence of a shifted LSPR peak using absorbance spectroscopy and the reaction was stopped (typically after 2 min) using $N$-ethylmaleimide. Examples of the type of dimers obtained in this way are shown in the transmission electron microscopy (TEM) images in Fig. 3. For TEM imaging, $5 \mu \mathrm{l}$ of the solution containing gold NP dimers was drop cast onto a TEM grid ( $3 \mathrm{~mm}$ diameter, 200 mesh amorphous holey carbon-coated copper grid (EM Resolu-

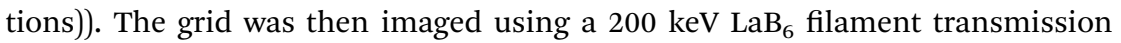
electron microscope (Tecnai T20, FEI) with a $4096 \times 4096$ pixel 16 bit bottommounted camera (BM Eagle, FEI). For optical measurements, solutions with gold NP dimers were covalently bound onto a glass coverslip functionalised with 3-MT, covered in silicone oil, and sealed with a glass slide. The solution contained a mixture of isolated NPs, dimers and larger aggregates (see overview TEM image Fig. 3a). We therefore investigated the applicability of our wide-field microscopy method to characterize such a heterogeneous ensemble, and specifically, to distinguish dimers from single particles and larger aggregates.

A cloud plot representing a combination of the extinction cross-section $\sigma_{\text {ext }}$ measured for these NPs on glass in the G and R channels (using the $40 \times 0.95$ NA objective, 1.5 magnifier and the Canon camera) alongside the asymmetry parameter $\alpha_{\mathrm{P}}$ (for $\sigma_{\text {sca }}$ ) in the corresponding channels is shown in Fig. 4. We chose

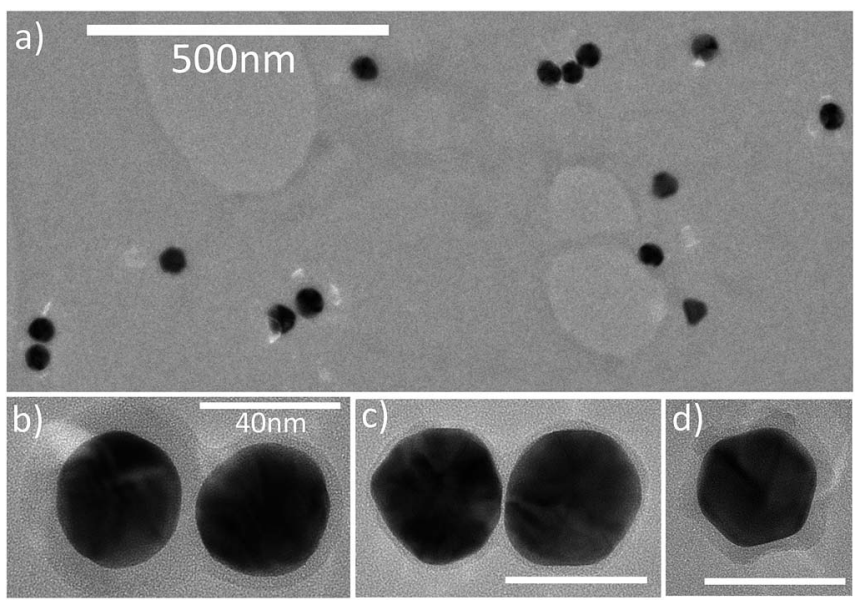

Fig. 3 Transmission electron microscopy (TEM) images of gold NPs drop-cast onto a TEM grid, from a solution containing nominal $40 \mathrm{~nm}$ NPs coated with a nominal $6 \mathrm{~nm}$ thick silica shell, covalently bound to bare $40 \mathrm{~nm}$ NPs. (a) Overview showing single particles, dimers and trimers. (b) Example of a dimer between a silica-coated NP (left) and a bare NP (right). (c) Example of a dimer between two bare NPs. (d) Example of a single $40 \mathrm{~nm} N \mathrm{NP}$ coated with a $6 \mathrm{~nm}$ silica shell. The thin coating visible on the nominally bare NPs is likely due to the (3-mercaptopropyl)trimethoxysilane forming a polymerized structure. 


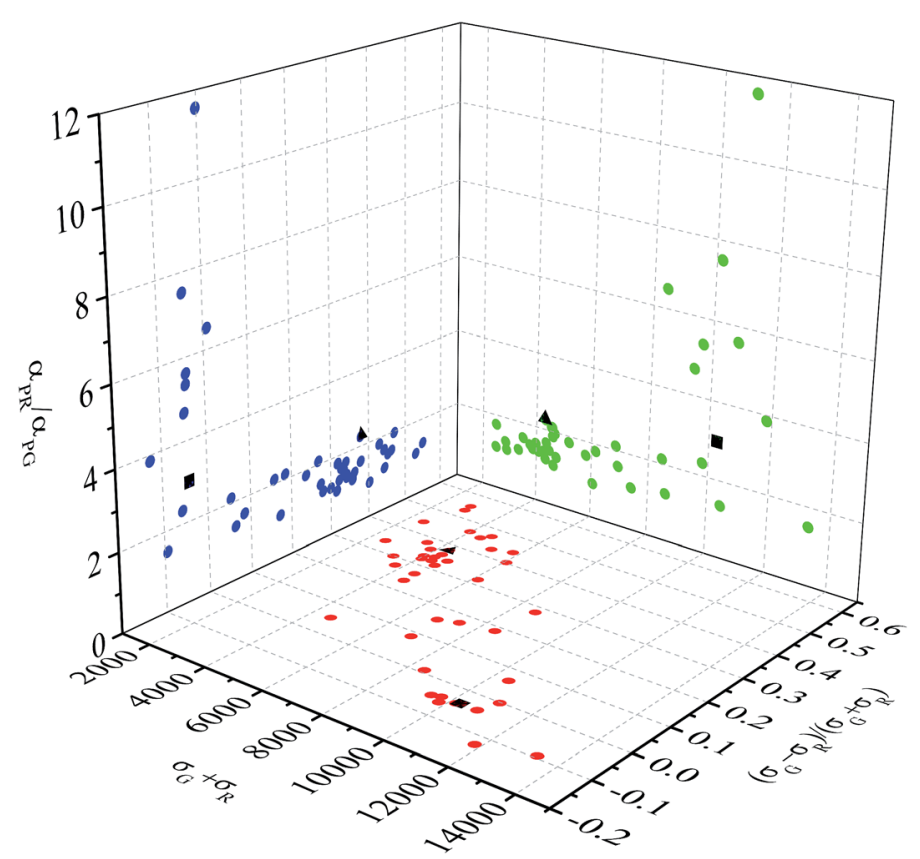

Fig. 4 Optical properties measured on individual NPs covalently bound onto glass, from a solution containing nominal $40 \mathrm{~nm}$ NPs coated with a $6 \mathrm{~nm}$ thick silica shell and covalently bound to bare $40 \mathrm{~nm}$ NPs (same as in Fig. 3). Measurements are shown as a trivariate distribution using the cross-section $\sigma_{R}+\sigma_{G}$, the contrast $\left(\sigma_{G}-\sigma_{R}\right) /\left(\sigma_{G}+\sigma_{R}\right)$, and the asymmetry parameter ratio $\alpha_{\mathrm{PR}} / \alpha_{\mathrm{PG}}$ (see text). Black symbols indicate the two particles shown in Fig. 5.

to represent $\alpha_{\mathrm{P}}$ extracted from $\sigma_{\text {sca }}$ due to its lower uncertainty compared to $\alpha_{\mathrm{P}}$ from $\sigma_{\text {ext }}$ when measuring with the Canon color camera. ${ }^{13}$ The coordinates were chosen as (i) the extinction cross-section represented by the sum of R and $\mathrm{G}$ channels $\sigma_{\mathrm{R}}+\sigma_{\mathrm{G}}$, (ii) the $\mathrm{G}$ to R cross-section contrast $\left(\sigma_{\mathrm{G}}-\sigma_{\mathrm{R}}\right) /\left(\sigma_{\mathrm{G}}+\sigma_{\mathrm{R}}\right)$, and (iii) the asymmetry ratio $\alpha_{\mathrm{PR}} / \alpha_{\mathrm{PG}}$. The cross-section indicates whether particles are single or aggregates, since this value will scale roughly proportional to the number of particles in the focal volume. The differential quantity $\left(\sigma_{\mathrm{G}}-\sigma_{\mathrm{R}}\right) /$ $\left(\sigma_{\mathrm{G}}+\sigma_{\mathrm{R}}\right)$ gives information on the spectral position of the LSPR, where we expect isolated spherical particles to have $\sigma_{\mathrm{G}}>\sigma_{\mathrm{R}}$, while dimers and aggregates will have $\sigma_{\mathrm{R}}>\sigma_{\mathrm{G}}$ due to the red shift of the LSPR upon plasmonic coupling in a dimer with a gap much smaller than the NP diameter. ${ }^{20}$ The ratio $\alpha_{\mathrm{PR}} / \alpha_{\mathrm{PG}}$ is used to emphasize the asymmetry due to dimer formation, where we expect $\alpha_{\mathrm{PR}} / \alpha_{\mathrm{PG}} \gtrsim 1$ for a single slightly aspherical particle and $\alpha_{\mathrm{PR}} / \alpha_{\mathrm{PG}} \gg 1$ for dimers with small gaps. We can see a systematic trend in Fig. 4 with isolated particles being the group where $\left(\sigma_{\mathrm{G}}-\sigma_{\mathrm{R}}\right) /\left(\sigma_{\mathrm{G}}+\sigma_{\mathrm{R}}\right)>0, \alpha_{\mathrm{PR}} / \alpha_{\mathrm{PG}}<2$ and $\sigma_{\mathrm{R}}+\sigma_{\mathrm{G}}<6000 \mathrm{~nm}^{2}\left(\bar{\sigma}_{\text {ext }}=4000 \mathrm{~nm}^{2}\right.$ in the $\mathrm{G}$ channel and $\bar{\sigma}_{\text {ext }}=2000 \mathrm{~nm}^{2}$ in the R channel for an isolated $40 \mathrm{~nm} \mathrm{NP}$ ), while strongly coupled dimers are being identified as the group where $\left(\sigma_{\mathrm{G}}-\sigma_{\mathrm{R}}\right)$ / $\left(\sigma_{\mathrm{G}}+\sigma_{\mathrm{R}}\right)<0, \alpha_{\mathrm{PR}} / \alpha_{\mathrm{PG}}>2$ and $12000>\sigma_{\mathrm{R}}+\sigma_{\mathrm{G}}>6000 \mathrm{~nm}^{2}$. For larger aggregates $\sigma_{\mathrm{R}}+\sigma_{\mathrm{G}}>12000 \mathrm{~nm}^{2},\left(\sigma_{\mathrm{G}}-\sigma_{\mathrm{R}}\right) /\left(\sigma_{\mathrm{G}}+\sigma_{\mathrm{R}}\right)<0$ but $\alpha_{\mathrm{PR}} / \alpha_{\mathrm{PG}}$ varies, depending on the anisotropy of the assembly. In fact, we see in the cloud plot a large aggregate with $\alpha_{\mathrm{PR}} / \alpha_{\mathrm{PG}}<2$ and $\sigma_{\mathrm{R}}+\sigma_{\mathrm{G}} \sim 14000 \mathrm{~nm}^{2}$, consistent with a closely-packed trimer. 
To confirm these attributions, for the two particles highlighted by black symbols in the cloud plot in Fig. 4, we have measured the spectrally-resolved and polarisation-resolved extinction. We inserted a linear polariser in the white-light illumination beam path before the condenser, and coupled the transmitted light collected by the microscope objective $(100 \times 1.45$ NA oil objective, $1 \times$ intermediate magnification) into an imaging spectrometer (Horiba Jobin-Yvon iHR 550) equipped with a 100 lines per mm grating (input slit opening of $60 \mu \mathrm{m}$ corresponding to $\sim 1 \mathrm{~nm}$ spectral resolution) and a CCD camera (Andor Newton DU-971N). Referencing was performed similarly to the extinction microscopy discussed in the previous section. Briefly, a stripe-like region of the sample containing a NP in the center was imaged onto the input slit of the spectrometer and, in turn, onto the CCD camera. Within this stripe, image regions above and below the NP were used as a reference under identical spectral illumination conditions. A measurement without the NP (by lateral shifting) was also acquired to correct for irregularities in the camera pixels. Fig. 5a shows the extinction spectra for the particle highlighted as a black triangle in Fig. 4. The spectra exhibit a single LSPR at around $540 \mathrm{~nm}$, almost independent of the polarisation angle, supporting the attribution that this particle is an isolate quasi-spherical NP. Fig. $5 \mathrm{~b}$ shows the extinction spectra for the particle highlighted as a black square in Fig. 4, which exhibit a strong polarisation dependence and two distinct LSPRs (one at $535 \mathrm{~nm}$ and the second one at $575 \mathrm{~nm}$ ) for two orthogonal polarisations, supporting the attribution that this particle is a dimer. Numerical simulations, using the software COMSOL Multiphysics, of the extinction spectra of a gold dimer surrounded by a homogeneous medium of refractive index 1.5, calculated for the two in-plane polarisations along and perpendicular to the interparticle axis, are shown for comparison (dotted lines in Fig. 5). The best agreement between simulations and the experiment is found assuming that the two NPs
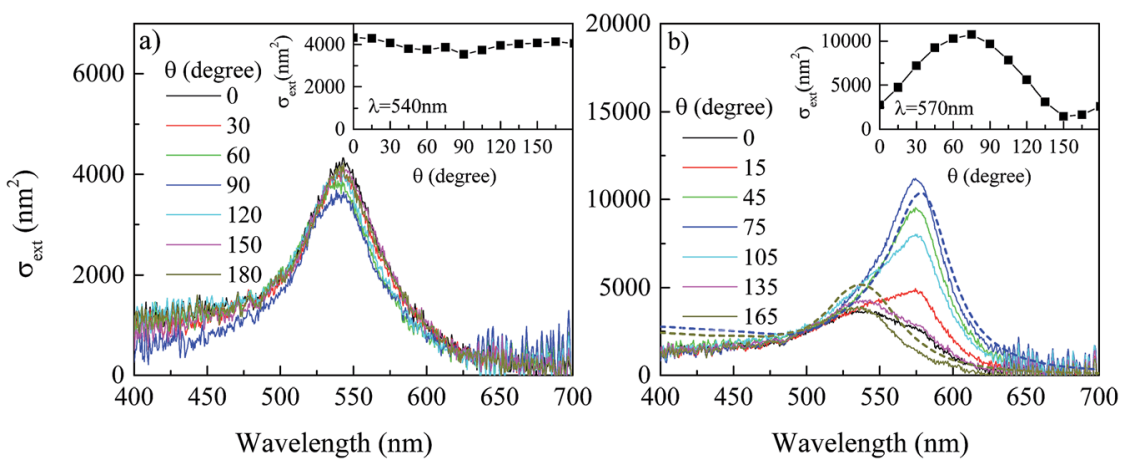

Fig. 5 Extinction spectra on the particles indicated as black symbols in Fig. 4 for various in-plane linear polarisation angles $\theta$ of the white-light illumination as indicated. The insets show the extinction versus polarizer angle at the indicated wavelength. (a) Spectra on the particle indicated by the black triangle in Fig. 4. (b) Spectra on the particle indicated by the black square in Fig. 4. Dotted lines are numerical simulations of the extinction spectra of a dimer consisting of a spherical NP of $32 \mathrm{~nm}$ diameter with a gap distance of $5.5 \mathrm{~nm}$ from a second particle of $33 \mathrm{~nm}$ diameter, calculated using COMSOL Multiphysics for the two in-plane polarisations along and perpendicular to the interparticle axis. Measured spectra were acquired using an exposure time of $0.1 \mathrm{~s}$, and 200 frames average. 
forming the dimer have slightly different diameters of $32 \mathrm{~nm}$ and $33 \mathrm{~nm}$ (heterodimer model) and an interparticle gap of $5.5 \mathrm{~nm}$. Larger diameters result in larger peak cross-sections, while different gaps do not reproduce the red-shifted LSPR wavelength at $575 \mathrm{~nm}$. Using NPs with different diameters enabled us to optimize the amplitude ratio between the longitudinal and transverse plasmon peaks. The deviation between simulations and experiment might be due to the imperfect sphericity of the particles, not included in the calculations. Furthermore, simulations assume an incident plane wave, and do not account for the experimental angular excitation and detection range.

\section{Resonant four-wave mixing microscopy}

Transient resonant four-wave mixing micro-spectroscopy was performed with a heterodyne interferometric detection very similar to the one discussed in our previous works, ${ }^{\mathbf{6} 14}$ the main difference being that we used an epi-detection geometry instead of a transmission geometry. Briefly, optical pulses of $\sim 100 \mathrm{fs}$ duration at $80 \mathrm{MHz}$ repetition rate and $550 \mathrm{~nm}$ center wavelength were provided by the signal output of an optical parametric oscillator (OPO). The pulse train is split into three beams (pump, probe and reference), having all the same center wavelength (degenerate FWM scheme). The pump beam excites the sample with an intensity which is temporally modulated by an acousto-optic modulator (AOM) driven with a square wave amplitude modulation of about $1 \mathrm{MHz}$ frequency. The change in the sample optical properties induced by this excitation is probed by the probe pulse at an adjustable delay time $\tau$ after the pump pulse. Pump and probe pulses of fields $E_{1}$ and $E_{2}$, respectively, are recombined into the same spatial mode and focused onto the sample by a microscope objective (for the data shown a $40 \times 0.95 \mathrm{NA}$ ) mounted into the same inverted microscope stand used for the extinction microscopy discussed in the previous section. The sample position is moved relative to the focal volume of the objective by an $x y z$ piezoelectric stage. A FWM signal (proportional to $E_{1} E_{1}^{*} E_{2}$ ) is collected in reflection (epi-direction) by the same objective, and recombined in a beam splitter with the reference pulse of adjustable delay. The resulting interference is detected by balanced photodiodes (for common-mode noise rejection) and a dual-channel high-frequency lock-in amplifier. We used a heterodyne scheme where the probe optical frequency is upshifted by a radio-frequency amount using an AOM and its interference with the unshifted reference is detected at the appropriate beat frequency, as in the scheme published in ref. 14. This enables us to discriminate the FWM field from the pump and probe pulses and to detect the amplitude and phase of the field. Full control of the pump, probe and reference polarisation was achieved using waveplates in each beam. Note that this interferometric time-resolved FWM detection enables background-free imaging of single gold NPs even in highly scattering and fluorescing environments. ${ }^{6}$

In Fig. 6a we first recall an example of FWM dynamics versus $\tau$ measured on a single NP of nominal $40 \mathrm{~nm}$ diameter drop cast onto glass (from ref. 14) alongside the theoretical model discussed in detail in ref. 14. A key point is the ability of the technique to distinguish the real and imaginary part of the pump-induced change in the particle complex polarisability, indicated as $\Delta \hat{\varepsilon}_{\mathrm{R}}$ and $\Delta \hat{\varepsilon}_{\mathrm{I}}$, respectively (where we defined an effective dielectric constant $\hat{\varepsilon}$ proportional to the NP polarisability $\alpha=4 \pi R^{3} \hat{\varepsilon}$ for spherical particles, and denoted the pump-induced 

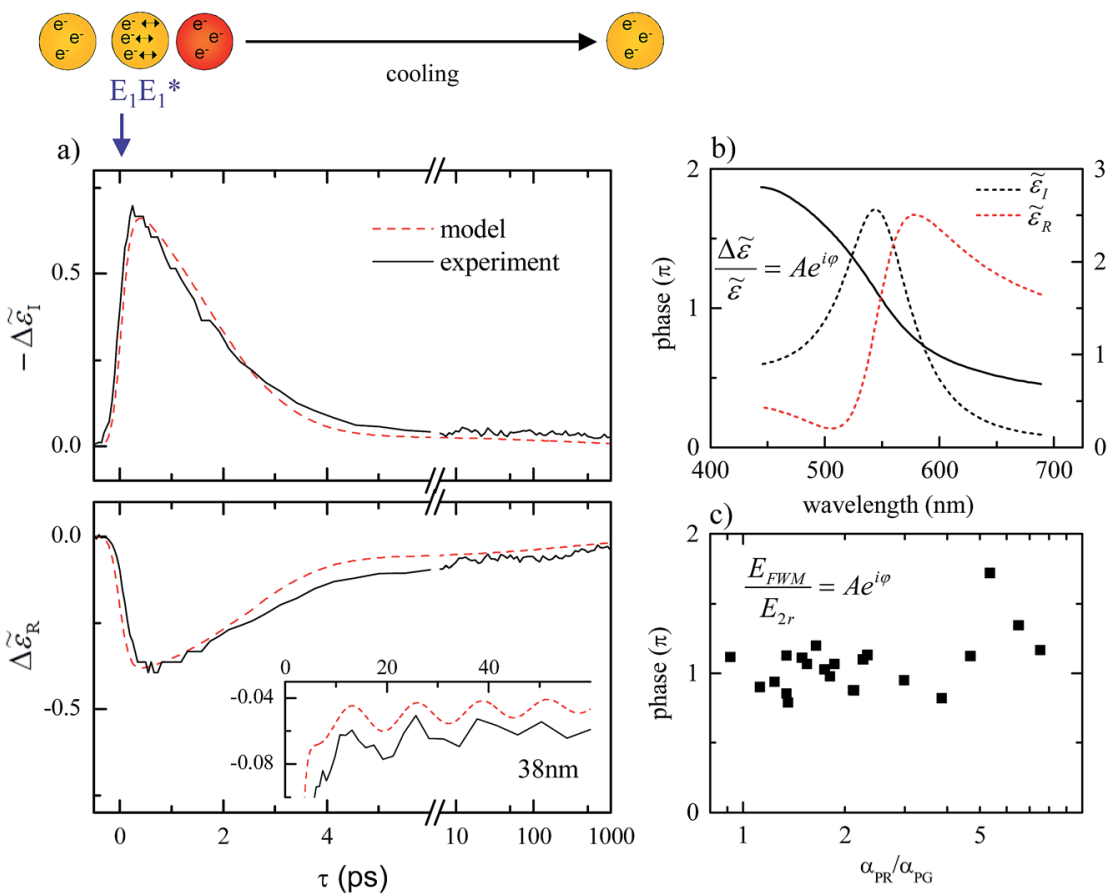

Fig. 6 a) Transient changes of the real $\left(\Delta \hat{\varepsilon}_{R}\right)$ and imaginary $\left(\Delta \hat{\varepsilon}_{1}\right)$ part of the dielectric function of a single $38 \mathrm{~nm}$ diameter gold NP resonantly excited and probed at $550 \mathrm{~nm}$. The pump (probe) fluence is $0.65 \mathrm{~J} \mathrm{~m}^{-2}\left(0.05 \mathrm{~J} \mathrm{~m}^{-2}\right)$. The acquisition time per point is $200 \mathrm{~ms}$. The dashed lines are the corresponding calculations. The top sketch illustrates the LSPR excitation by the pump $\left(E_{1}\right)$ and the subsequent heating and cooling dynamics monitored by the probe pulse. The inset shows coherent phonon oscillations from which the $38 \mathrm{~nm}$ diameter is deduced (reproduced from ref. 14). (b) Spectrally resolved real and imaginary part of the dielectric constant $\hat{\varepsilon}$ (dotted lines) simulated for the $38 \mathrm{~nm}$ NP in (a) prior to the arrival of the pump, together with the phase $\varphi$ (solid line) of the complex quantity $\Delta \hat{\varepsilon} / \hat{\varepsilon}$ with the pump-induced change $\Delta \hat{\varepsilon}$ calculated at $\tau=0.5 \mathrm{ps}$. (c) Measured phase of the ratio between the FWM field and the reflected probe at $\tau=0.5 \mathrm{ps}$ on a group of NPs from the cloud plot in Fig. 4 as a function of the asymmetry parameter $\alpha_{\mathrm{PR}} / \alpha_{\mathrm{PG}}$.

change as $\Delta \hat{\varepsilon})$. Both $\Delta \hat{\varepsilon}_{\mathrm{R}}$ and $\Delta \hat{\varepsilon}_{\mathrm{I}}$ exhibit dynamics that are quantitatively explained by the model. These are due to the pump-induced increase in the electron temperature (which, in turn, broadens and shifts the LSPR on a $\sim 100$ fs time scale), followed by a recovery to equilibrium via the thermalization of the hot electron gas with the cooler lattice by electron-phonon coupling ( $\sim 1$ ps time scale), and the thermalization of the heated NP with the cooler surroundings ( $\sim 100$ ps time scale). We also observe coherent phonon oscillations in $\Delta \hat{\varepsilon}_{\mathrm{R}}$ (see inset in Fig. 6a). These are due to the modulation of the plasma frequency by the breathing vibrational mode of the NP with an oscillation period proportional to the particle radius ${ }^{21-23}$ from which we infer a diameter of $38 \mathrm{~nm}$ for the specific NP shown.

Besides these dynamics, we showed in ref. 14 that the phase of $\Delta \hat{\varepsilon}$ is sensitive to the spectral position of the LSPR relative to the probe optical frequency in the experiment. The phase is a ratiometric quantity independent of the signal 
strength, and therefore could be used for robust LSPR-based sensing applications. Owing to the red-shift of the LSPR occurring in a NP dimer (increasing with decreasing interparticle gap distance ${ }^{20}$ ), we would thus expect that this phase measured at $550 \mathrm{~nm}$ wavelength in our FWM experiment would gradually change, going from a single quasi-spherical NP to a plasmonically coupled dimer (in a gap-dependent manner). To address this expectation, we measured such phase on the group of NPs identified as single quasi-spherical or dimers in Fig. 4.

Since the experiment is performed in epi-geometry, we are detecting the complex quantity $\Delta \hat{\varepsilon} / \hat{\varepsilon}$ via the ratio between the FWM field $E_{\mathrm{FWM}} \propto \Delta \hat{\varepsilon} E_{2}$ and the reflected probe field $E_{2 r} \propto \hat{\varepsilon} E_{2}$ (as opposed to the transmitted probe field $E_{2}$ in ref. 14). The phase $\varphi$ of $\Delta \hat{\varepsilon} / \hat{\varepsilon}$ calculated from $\Delta \hat{\varepsilon}$ at $\tau=0.5$ ps for a spherical $40 \mathrm{~nm} \mathrm{NP}$ (see Fig. 5 in ref. 14) is plotted in Fig. $6 \mathrm{~b}$ together with the real and imaginary parts of $\hat{\varepsilon}$. From this calculation, we expect to observe $\varphi \sim \pi$ for a NP at resonance and $\varphi$ $>\pi$ when probing at shorter wavelengths compared to the LSPR. As shown in Fig. 5, the LSPR of the coupled (longitudinal) plasmon mode in a dimer is significantly red shifted compared to the wavelength at $550 \mathrm{~nm}$ used in the FWM experiment. Hence, based on these qualitative considerations, we would expect to observe $\varphi>\pi$ in a plasmonically coupled dimer compared to $\varphi \sim \pi$ in a single spherical NP. Fig. $6 \mathrm{c}$ shows the measured phase of the ratio $E_{\mathrm{FWM}} / E_{2 r}$ in a group of NPs from the plot in Fig. 4, as a function of the asymmetry ratio $\alpha_{\mathrm{PR}} / \alpha_{\mathrm{PG}}$. Measurements were performed using a circularly polarized pump and probe and a co-circularly polarized reference, rather than linear polarisation as in ref. 14, to enable the excitation of dimers without prior knowledge of their orientation. We observe that NPs with a large asymmetry parameter ratio $\alpha_{\mathrm{PR}} / \alpha_{\mathrm{PG}}>2$, indicating dimers according to Fig. 4, tend to exhibit $\varphi>\pi$ compared to single quasispherical NPs with $\alpha_{\mathrm{PR}} / \alpha_{\mathrm{PG}}<2$ and $\varphi \sim \pi$, in agreement with our qualitative expectation. A more in-depth understanding of the measured phase both experimentally and theoretically, including a careful calibration against instrumentrelated phase offsets in the set-up, ${ }^{14}$ a confirmation that the measured particles are dimers via correlative TEM, and a more quantitative description of the expected dimer response versus interparticle distance and polarisation configuration is in progress and will be published in a future manuscript.

\section{Conclusions}

In conclusion, we have developed a rapid and sensitive method to quantitatively measure the optical extinction cross-section of individual nanoparticles using wide-field imaging. The method enables simultaneous acquisition of hundreds of nanoparticles for statistical analysis with a sensitivity limit down to $5 \mathrm{~nm}^{2}$, corresponding to a single $5 \mathrm{~nm}$ gold $\mathrm{NP}$, using acquisition times in the $1 \mathrm{~min}$ range. An experimental demonstration is shown on single nominally-spherical gold nanoparticles with diameters ranging from $10 \mathrm{~nm}$ to $100 \mathrm{~nm}$. Importantly, the method can be implemented cost-effectively on any conventional wide-field microscope and is applicable to any nanoparticle. Furthermore, we have investigated the effect of the particle asphericity on the optical extinction cross-section measured with in-plane linearly polarised light as a function of the polarisation direction, and derived, from theory, a simple expression linking these measurements to the particle aspect ratio. We also fabricated gold NP dimers and showed that the optical extinction cross-section analysis can be used to distinguish 
plasmonically-coupled dimers from single quasi-spherical NPs and larger aggregates. Finally, we performed transient resonant FWM heterodyne interferometry on the same group of NPs characterized by the optical extinction cross-section analysis. We specifically investigated the phase of the ratio between the FWM field and reflected probe field, which is expected to be sensitive to the relative shift between the nanoparticle LSPR and the optical probe wavelength. A comparison between single NPs and dimers identified from the optical extinction analysis supported this expectation and highlighted the potential of these phase-resolved measurements for LSPR-based sensing applications.

\section{Data access}

Data access Information about the data created during this research, including how to access it, is available from Cardiff University data archive at http:// dx.doi.org/10.17035/d.2015.100095.

\section{Acknowledgements}

This work was funded by the EPSRC UK Research Council under the grant agreement EP/L001470/1. P.B. acknowledges the EPSRC for her Leadership fellowship award EP/I005072/1 and the Royal Society for her Wolfson Research Merit Award. W.L. acknowledges support by a Leverhulme Royal Society Research Fellowship (Grant no. LT20085).

\section{References}

1 A. Crut, P. Maioli, N. D. Fatti and F. Vallée, Chem. Soc. Rev., 2014, 43, 39213956.

2 W. Barnes, A. Deraux and T. Ebbesen, Nature, 2003, 424, 824-830.

3 K. Awazu, M. Fujimaki, C. Rockstuhl, J. Tominaga, H. Murakami, Y. Ohki, N. Yoshida and T. Watanabe, J. Am. Chem. Soc., 2008, 130, 1676-1680.

4 H. Atwater and A. Polman, Nat. Mater., 2010, 9, 205-213.

5 L. Cognet, S. Berciaud, D. Lasne and B. Lounis, Anal. Chem., 2008, 80, 22882294.

6 F. Masia, W. Langbein, P. Watson and P. Borri, Opt. Lett., 2009, 34, 1816-1818.

7 K. Kneipp, H. Kneipp, I. Itzkan, R. Dasari and M. Feld, J. Phys.: Condens. Matter, 2002, 14, R597-R624.

8 A. McFarland and R. Van Duyne, Nano Lett., 2003, 3, 1057-1062.

9 C. Sönnichsen, B. M. Reinhard, J. Liphardt and A. P. Alivisatos, Nat. Biotechnol., 2005, 23, 741-745.

10 C. Ayala-Orozco, C. Urban, M. W. Knight, A. S. Urban, O. Neumann, S. W. Bishnoi, S. Mukherjee, A. M. Goodman, H. Charron, T. Mitchell, M. Shea, R. Roy, S. Nanda, R. Schiff, N. J. Halas and A. Joshi, ACS Nano, 2014, 8, 6372-6381.

11 A. Arbouet, D. Christofilos, N. D. Fatti, F. Vallée, J. R. Huntzinger, L. Arnaud, P. Billaud and M. Broyer, Phys. Rev. Lett., 2004, 93, 127401.

12 D. Boyer, P. Tamarat, A. Maali, B. Lounis and M. Orrit, Science, 2002, 297, 1160-1163.

13 L. M. Payne, W. Langbein and P. Borri, Appl. Phys. Lett., 2013, 102, 131107. 
14 F. Masia, W. Langbein and P. Borri, Phys. Rev. B: Condens. Matter Mater. Phys., 2012, 85, 235403.

15 M. van Dijk, A. Tchebotareva, M. Orrit, M. Lippitz, S. Berciaud, D. Lasne, L. Cognet and B. Lounis, Phys. Chem. Chem. Phys., 2006, 8, 3486-3495.

16 O. L. Muskens, P. Billaud, M. Broyer, N. D. Fatti and F. Vallée, Phys. Rev. B: Condens. Matter Mater. Phys., 2008, 78, 205410.

17 A. Tcherniak, J. Ha, S. Dominguez-Medina, L. Slaughter and S. Link, Nano Lett., 2010, 10, 1398-1404.

18 I. Pope, L. Payne, G. Zoriniants, E. Thomas, O. Williams, P. Watson, W. Langbein and P. Borri, Nat. Nanotechnol., 2014, 9, 940-946.

19 C. F. Bohren and D. R. Huffman, Absorption and Scattering of Light by Small Particles, Wiley-VCH, 1998.

20 P. K. Jain, W. Huang and M. A. El-Sayed, Nano Lett., 2007, 7, 2080-2088.

21 C. Voisin, N. D. Fatti, D. Christofilos and F. Vallee, J. Phys. Chem. B, 2001, 105, 2264-2280.

22 M. A. van Dijk, M. Lippitz and M. Orrit, Phys. Rev. Lett., 2005, 95, 267406.

23 G. V. Hartland, Chem. Rev., 2011, 111, 3858-3887. 